\title{
The Effects of Bilirubin on Brain Energy Metabolism during Hyperosmolar Opening of the Blood-Brain Barrier: An In Vivo Study Using ${ }^{31} \mathrm{P}$ Nuclear Magnetic Resonance Spectroscopy
}

\author{
N. K. IVES, N. M. BOLAS, AND R. M. GARDINER \\ Department of Paediatrics and MRC Biomedical NMR Group, John Radcliffe Hospital, Headington, \\ Oxford, United Kingdom
}

\begin{abstract}
Despite intensive investigation it remains uncertain how bilirubin enters the brain and how it exerts a toxic effect on neurones. Studies of induced hyperbilirubinemia in animal models in vivo have failed to reproduce bilirubin encephalopathy without additional factors such as hypoxia, asphyxia, hypercapnia, and disruption of the blood-brain barrier. The aim of this study was to investigate, using ${ }^{31} \mathbf{P}$ NMRS, whether hyperbilirubinemia alone or in association with hyperosmolar opening of the bloodbrain barrier caused any disturbance of cerebral energy metabolism in vivo. Spectra were acquired using a surface coil positioned over the right cerebral hemisphere of anaesthetized adult rats placed in the bore of a 1.9 Tesla magnet. Hyperbilirubinemia alone at a maximum mean serum concentration of $1063 \pm 175 \mu \mathrm{mol} / \mathrm{L}$ (mean $\pm \mathrm{SD}$, $n=7$ ) caused no apparent disruption in brain energy metabolism. However, in combination with hyperosmolar blood-brain barrier opening a serum bilirubin concentration of $483 \pm 52 \mu \mathrm{mol} / \mathrm{L}$ (mean $\pm \mathrm{SD}, n=9$ ) was associated with a reduction in $\mathrm{PCr} /(\mathrm{PCr}+\mathrm{Pi})$ ratio from $0.68 \pm 0.06$ to $0.44 \pm 0.14$ (mean $\pm \mathrm{SD}, p<0.001$ ). A significant correlation was demonstrated between cerebral hemisphere bilirubin content and the reduction in $\mathrm{PCr} /(\mathrm{PCr}+\mathrm{Pi})(r=$ $0.84, n=9, p<0.01$ ). These results demonstrate in vivo a disruptive effect of bilirubin on cerebral energy metabolism in the presence of an open BBB. This mode of entry and mechanism of toxicity may be factors in the pathophysiology of bilirubin encephalopathy in the newborn infant. (Pediatr Res 26: 356-361, 1989)
\end{abstract}

\section{Abbreviations}

BBB, blood-brain barrier

PCr, phosphocreatine

pHi, intracellular pH

$\mathrm{Pi}$, inorganic phosphate

${ }^{31} \mathrm{P}$ NMRS, ${ }^{31}$ phosphorus nuclear magnetic resonance spectroscopy

The neurotoxicity of bilirubin in the newborn was first recognized in relation to the neurologic sequelae of severe jaundice associated with hemolytic disease in term infants. Although

Received January 13, 1989; accepted May 12, 1989.

Correspondence Dr. N. K. Ives, Department of Paediatrics, University of Oxford, John Radcliffe Hospital, Headington, Oxford OX3 9DU, UK.

Supported by the Medical Research Council, UK and The National Fund for Research into Crippling Diseases. severe jaundice due to this cause is now rare, the potential neurotoxic effects of bilirubin remain a common and important concern in the care of the newborn. Jaundice is a common occurrence in low birth weight infants, and frequently coexists with hypoxia or other events that may exacerbate bilirubin neurotoxicity. In addition, there is concern that milder degrees of jaundice, hitherto considered safe, may be associated with transient or even permanent CNS sequelae.

Despite intensive investigation it remains uncertain how bilirubin enters the brain and how it exerts a toxic effect on neurones. These two questions are central to our understanding of the pathophysiology of bilirubin encephalopathy, but have proved exceptionally difficult to address. Investigation has been hindered by the complexity of the biochemical behavior of the bilirubin molecule, and the lack of methods for measuring relevant variables in the newborn infant. Inevitable uncertainties arise in extrapolating from the animal models and in vitro techniques that investigators have been obliged to utilize.

Animal studies of induced hyperbilirubinemia have failed to reproduce bilirubin encephalopathy in the absence of additional factors such as hypoxia, asphyxia or hypercapnia $(1-3)$. Several recent animal studies have shown that hyperbilirubinemia in association with hyperosmolar opening of the BBB is associated with bilirubin entry into the brain $(4,5)$, and disturbed neuronal function as measured by electrophysiologic techniques (6). A substantial body of evidence in vitro supports the contention that interference with mitochondrial function is one component of the toxic effect of bilirubin on neurones $(7,8)$. The advent of phosphorus magnetic resonance spectroscopy provides the opportunity for evaluation of the effects of bilirubin on brain energy metabolism in vivo.

Preliminary observations indicated that hyperbilirubinemia in the adult rat was not associated with any disturbance of brain energy metabolism detectable by ${ }^{31} \mathrm{P}$ NMR spectra from the cerebral hemispheres. The aim of the present experiments was to determine whether hyperbilirubinemia in association with hyperosmolar opening of the BBB caused any derangement of cerebral hemisphere energy metabolism in vivo using ${ }^{31} \mathrm{P}$ magnetic resonance spectroscopy.

\section{MATERIALS AND METHODS}

Surgical procedure. Studies were performed on 23 adult male Wistar rats (300-500 g body wt) under halothane anaesthesia (Fluothane, IC, Macclesfield, England). Catheters were placed in the left femoral vein and artery for infusion of bilirubin and albumin solutions, and blood sampling. Tracheostomy was performed and ventilation instituted to maintain normal respiratory blood gas tensions. The right external carotid artery was retrogradely cannulated with polythene tubing (Portex, 3FG, Portex, 
Hythe, England) such that the tip was just distal to the bifurcation of the common carotid artery. The skull was exposed and temporoparietal muscles removed to ensure that the signal detected by the surface radiofrequency coil originated from the brain. The animal was supported on a warmed platform to maintain normal body temperature. Arterial blood pressure was monitored continuously, and respiratory blood gas tensions were estimated at regular intervals.

Materials. A solution of bilirubin $(5.2 \mathrm{mM})$ and albumin $(400$ $\mu \mathrm{M}$ ) was prepared under conditions of subdued light immediately before use. Crystalline bilirubin, $16 \mathrm{mg}$ (Sigma Chemical Co., St. Louis, MO) was dissolved in $1 \mathrm{~mL} 0.5 \mathrm{M} \mathrm{NaOH}$, and made up to $2 \mathrm{~mL}$ with distilled $\mathrm{H}_{2} \mathrm{O}$. BSA (Sigma), $8 \mathrm{~mL}$ of a $500 \mu \mathrm{M}$ solution, was added and the $\mathrm{pH}$ adjusted to 8.5 with $1 \mathrm{M} \mathrm{HCl}$. Bilirubin was omitted from an otherwise identical solution to provide a control infusion. A $1.8 \mathrm{M}$ solution of arabinose was prepared and warmed to $37^{\circ} \mathrm{C}$ before unilateral carotid perfusion.

Biochemical estimations. Serum bilirubin concentrations were estimated by spectrophotometry using an American Optical (Buffalo, NY) bilirubinometer with sample dilution as necessary. Right and left cerebral hemisphere bilirubin content was determined after extraction from homogenates with chloroform as described by Sawasaki et al. (9). Correction for intravascular bilirubin was made using a value for the vascular space of brain of $0.041 \mathrm{~mL} / \mathrm{g}(10)$, and the measured serum bilirubin concentrations at the end of the experiment.

NMR spectroscopy. ${ }^{31} \mathrm{P}$ NMR spectra were acquired using a Bruker Biospec spectrometer (Abingdon, Oxford, England) interfaced to an Oxford Research Systems 1.9 Tesla magnet (Abingdon), operating at a frequency of $32.5 \mathrm{MHz}$ for phosphorus. A 2-cm diameter, two-turn surface coil shaped to fit over the right cerebral hemisphere was applied to the exposed cranium. The coil was tuned and matched with a balanced circuit, and 128 or 256 transients were accumulated with an interpulse delay of $2 \mathrm{~s}$ and a pulse length chosen to give maximum signal to noise ratio. Free induction decays were deconvoluted by "profile correction" (second line broadenings 400, 200, factors 4, 2, respectively) followed by an exponential line broadening of $15 \mathrm{~Hz}$ and Fourier transformation. Peak areas were measured by triangulation.

Brain pHi was calculated from the chemical shift difference (ppm) between the Pi and PCr peaks in the ${ }^{31} \mathrm{P}$ spectrum using the equation

$$
\mathrm{pH}=6.75+\log \frac{(\delta-3.29)}{(5.69-\delta)}
$$

where $\delta=$ chemical shift between Pi and PCr peaks (11).

Experimental procedures. Bilirubin and albumin solutions (10 $\mathrm{mL}$ ) were infused intravenously over a 4-min period. Arabinose $(1.8 \mathrm{M})$ was administered via the right external carotid artery over $20 \mathrm{~s}$ at a rate of $7.5 \mathrm{~mL} / \mathrm{min}$. Arterial blood was sampled

Table 1. Experimental design

\begin{tabular}{llll}
\hline & \multicolumn{1}{c}{ Systemic infusion } & Unilateral carotid perfusion \\
& $(10 \mathrm{~mL}$ over $4 \mathrm{~min})$ & $(7.5 \mathrm{~mL} / \mathrm{min}$ for $20 \mathrm{~s})$ & $n$ \\
\hline 1 & Bilirubin $(5.2 \mathrm{mM})+\mathrm{BSA}(400 \mu \mathrm{M})$ & & 3 \\
2 & BSA $(400 \mu \mathrm{M})$ & Arabinose $(1.8 \mathrm{M})$ & 8 \\
3 & Bilirubin $(5.2 \mathrm{mM})+\mathrm{BSA}(400 \mu \mathrm{M})$ & Arabinose $(1.8 \mathrm{M})$ & 9 \\
4 & Isotonic saline or arabinose & 3 \\
\hline
\end{tabular}

for $\mathrm{pH}$ and blood gas tensions during a control period, after infusion of bilirubin or albumin solutions, before and after BBB opening, and at the end of each experiment. When bilirubin had been infused serum bilirubin estimations were made on the same blood samples. At the end of each experiment the cerebral hemispheres were dissected out and stored at $-20^{\circ} \mathrm{C}$ for subsequent bilirubin assay.

Experimental design. Each experiment was preceded by a period of 20 min during which two baseline ${ }^{31} \mathrm{P}$ NMR spectra were obtained $(256 \times 2$ transients). Four groups of experiments were then performed consisting of combinations of systemic intravenous infusion and unilateral carotid perfusion as listed in Table 1. In the first group of experiments the effect of hyperbilirubinemia alone was studied by acquiring up to 7 spectra $(128 \times 4256 \times 3$ transients $)$ over a period of $60 \mathrm{~min}$ after systemic infusion of bilirubin. The second group of experiments provided a control for hyperosmolar opening after systemic infusion of BSA. Spectra were acquired after BSA infusion (128 $\times 2$ transients) and after BBB opening $(128 \times 2,256 \times 2$ transients). The effect of BBB opening in the presence of hyperbilirubinemia was assessed in the third group of experiments. These differed from group 2 experiments only in the substitution of bilirubin for the BSA infusion. Group 4 consisted of control experiments in which systemic infusion was omitted and unilateral carotid perfusion was performed using isotonic saline, followed $30 \mathrm{~min}$ later by isotonic arabinose. Four spectra were collected after each perfusion $(128 \times 2256 \times 2$ transients $)$.

Statistics. Statistical significance was determined by analysis of variance and Student's $t$ test.

\section{RESULTS}

Throughout the studies described the animals' mean arterial blood pressure, blood gas tensions, and $\mathrm{pH}$ remained within the physiologic range and did not alter significantly with experimental manipulations (Table 2). After systemic infusion of bilirubin there was no apparent change in cerebral energy status [as judged by the ratio $\mathrm{PCr} /(\mathrm{PCr}+\mathrm{Pi})]$ in three animals studied for a period of $60 \mathrm{~min}$ (Fig. 1). The serum bilirubin concentrations, mean (range), were $1078 \mu \mathrm{mol} / \mathrm{L}$ (984-1200), $445 \mu \mathrm{mol} / \mathrm{L}(394-510)$, and $228 \mu \mathrm{mol} / \mathrm{L}(227-349)$ at 10,20 , and $55 \mathrm{~min}$, respectively, after the start of the bilirubin infusion. Similarly, no apparent changes were observed in nine animals during a $15-\mathrm{min}$ period of hyperbilirubinemia before BBB opening (Fig. 2). In these experiments the maximum recorded serum bilirubin concentration at $10 \mathrm{~min}$ after starting the infusion was $1063 \pm 175 \mu \mathrm{mol} /$ $\mathrm{L}$ (mean $\pm \mathrm{SD}, n=7$ ).

Infusion of the control albumin solution was not accompanied by any detectable change in the spectrum, but when followed by $\mathrm{BBB}$ opening there was a small fall in $\mathrm{PCr} /(\mathrm{PCr}+\mathrm{Pi})$ from 0.76 \pm 0.04 to $0.63 \pm 0.12$ (mean $\pm \mathrm{SD}, p<0.001, n=16$ ), as shown in Figure 2. $\mathrm{BBB}$ opening in the presence of hyperbilirubinemia was associated with a more marked disruption in brain energy status. The mean results of nine such experiments are given in Figure 2, and demonstrate a significant fall in $\mathrm{PCr} / \mathrm{PCr}+\mathrm{Pi}$ from $0.68 \pm 0.06$ to $0.44 \pm 0.14$ (mean $\pm \mathrm{SD}, p<0.001, n=$ 18). The mean serum bilirubin concentration at the time of BBB opening was $483 \pm 52 \mu \mathrm{mol} / \mathrm{L}$ (mean $\pm \mathrm{SD}, n=9$ ). An example of the effect of $\mathrm{BBB}$ opening in the presence of hyperbilirubinemia is shown as a representative sequence of spectra in Figure 3.

Table 2. Respiratory blood gas tensions, $p H$, and mean arterial blood pressure during experiments (mean $\pm S D)$

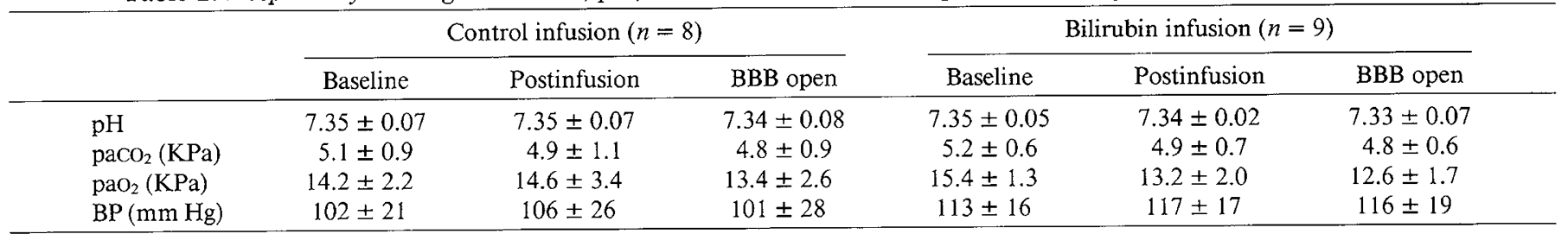


$\mathrm{PCr} /(\mathrm{PCr}+\mathrm{Pi})$

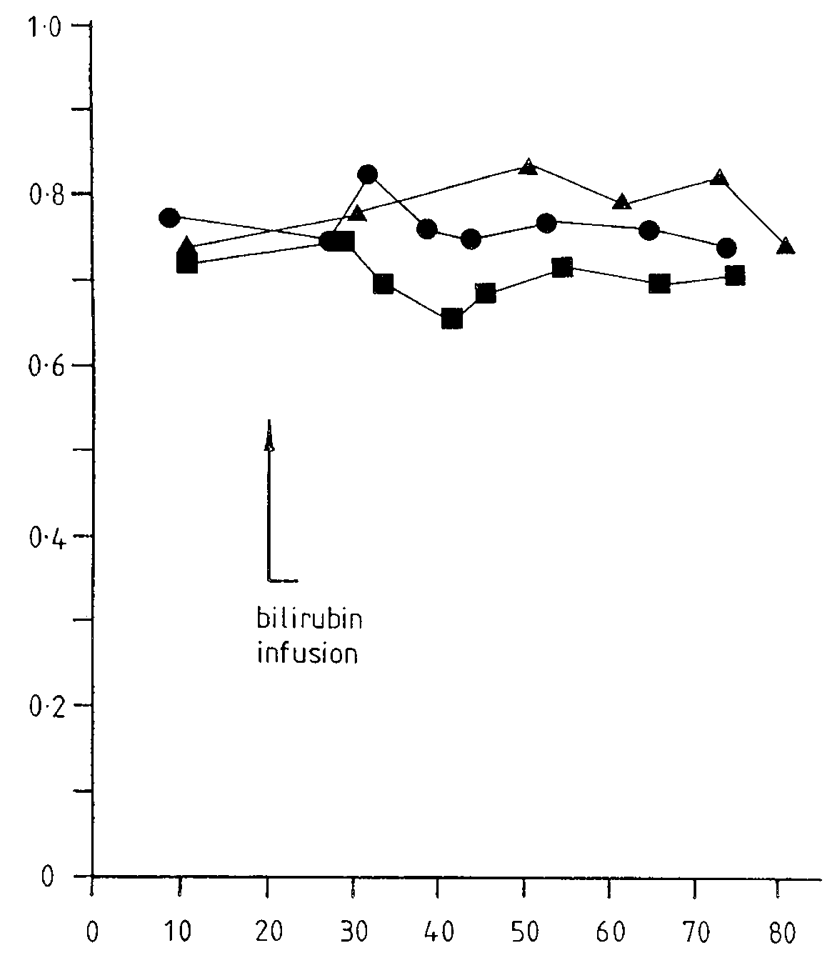

Minutes

Fig. 1. The effects of hyperbilirubinemia on $\mathrm{PCr} /(\mathrm{PCr}+\mathrm{Pi})(\mathrm{y}$ axis) in three rats studied for $60 \mathrm{~min}$ ( $\mathrm{x}$ axis) after bilirubin infusion.

$$
P C r /(P C r+P i)
$$

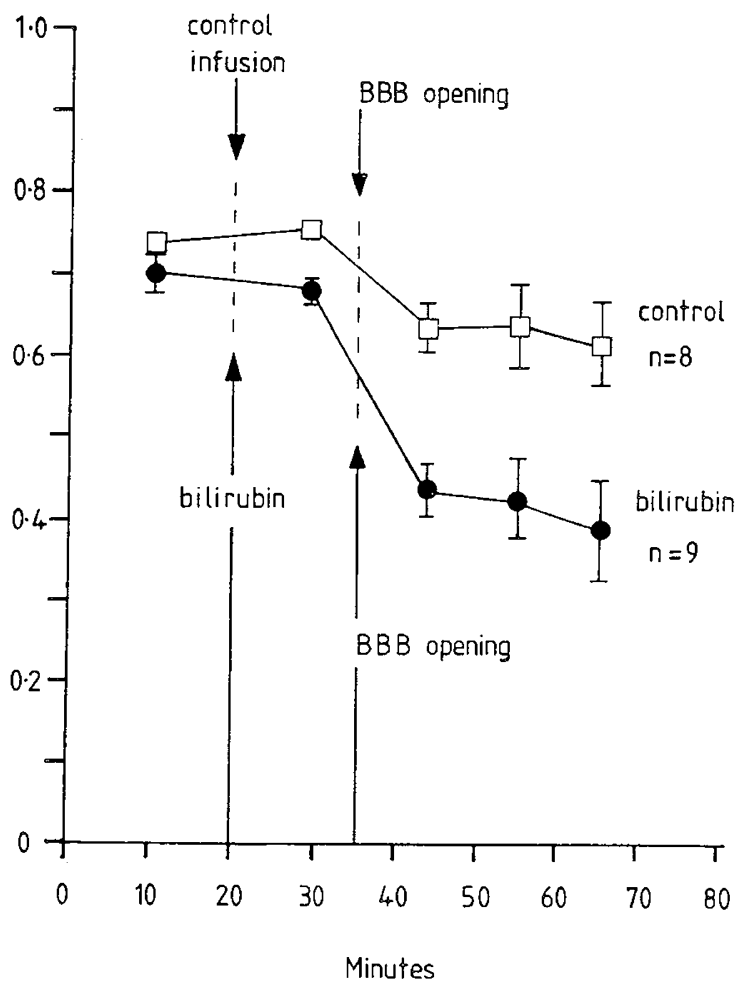

Fig. 2. Changes in $\mathrm{PCr} /(\mathrm{PCr}+\mathrm{Pi})$ (y axis) with bilirubin and control infusions before and after hyperosmolar opening of the BBB. Results expressed as mean $\pm \mathrm{SD}$. The values obtained immediately before and after $\mathrm{BBB}$ opening represent the mean value derived from two spectra for each experiment (128 transients $\times 2)$. The other values are the mean of single spectra derived from 256 transients.

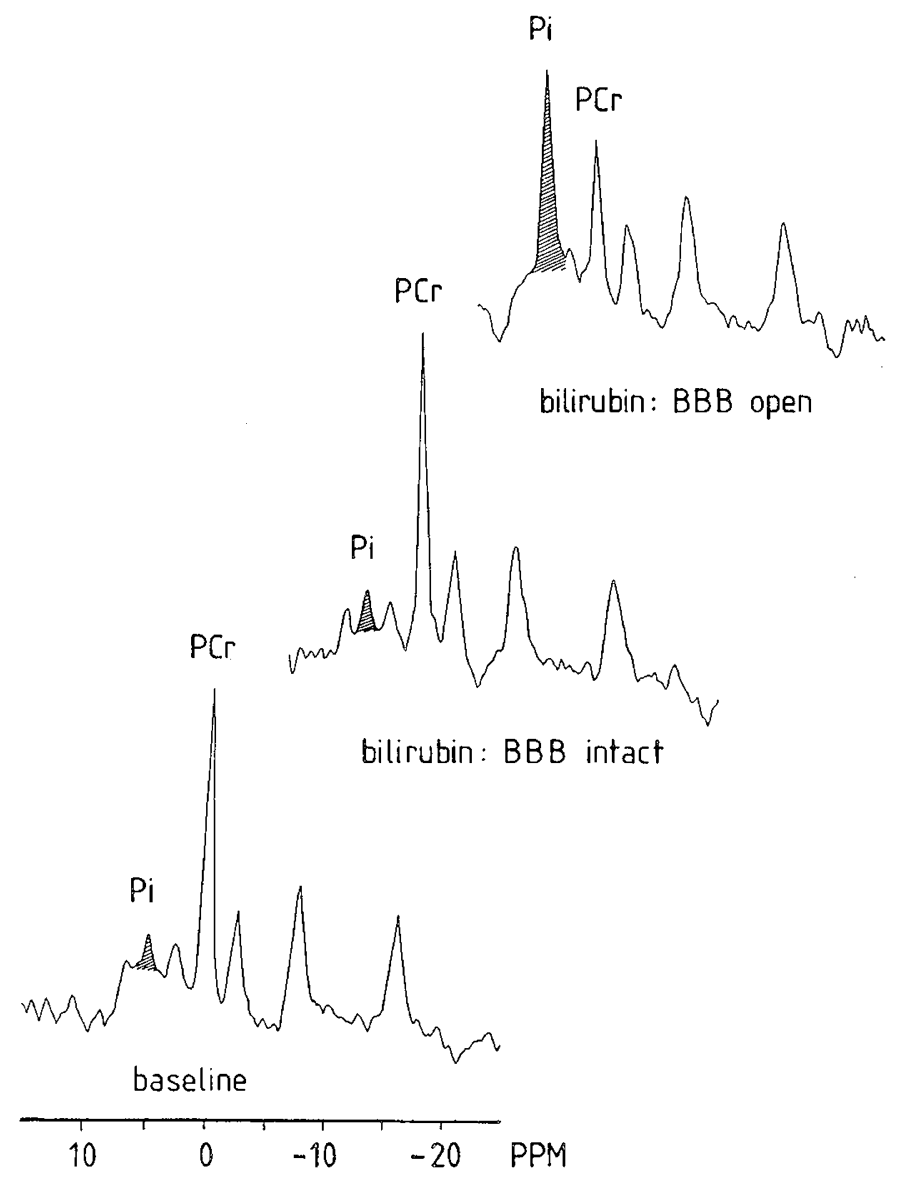

Fig. 3. A representative sequence of spectra from an individual rat under the experimental conditions as labeled. Hyperbilirubinemia caused no change in the control spectrum until the BBB was disrupted. Marked reciprocal changes in $\mathrm{Pi}$ and $\mathrm{PCr}$ were then observed. No significant changes occurred in the nucleoside triphosphate peaks.

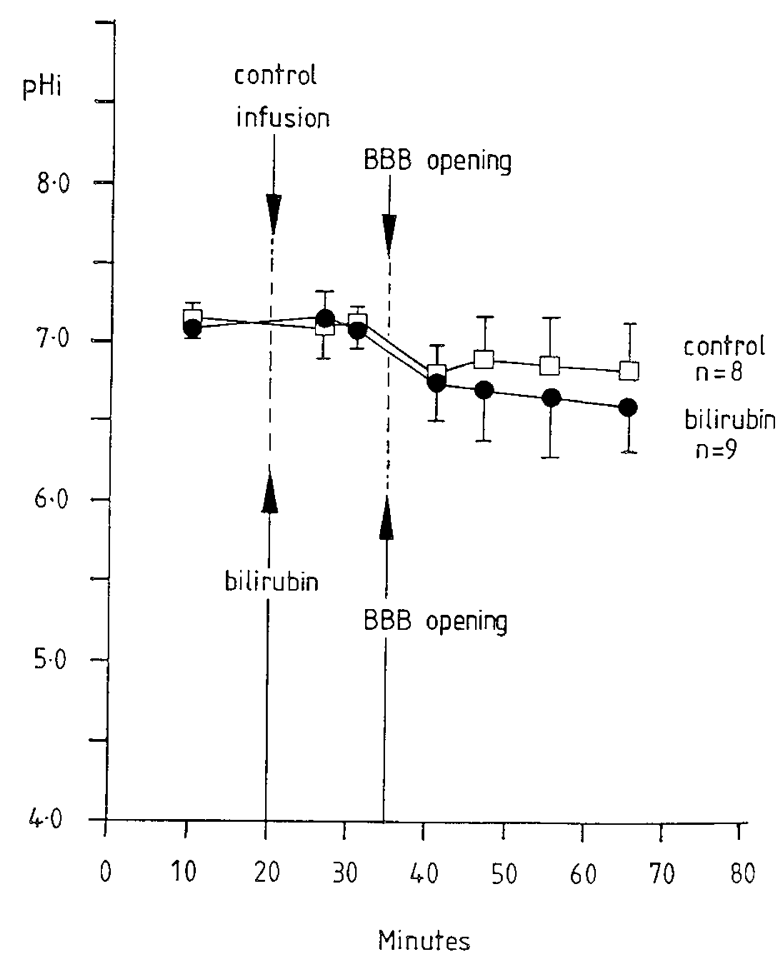

Fig. 4. Changes in brain $\mathrm{pHi}(\mathrm{y}$ axis) after bilirubin or control infusions and hyperosmolar opening. 
The fall in $\mathrm{PCr} /(\mathrm{PCr}+\mathrm{Pi})$ seen with hyperbilirubinemia in association with $\mathrm{BBB}$ opening was significantly greater than that observed with opening after the control albumin solution (interaction term, $p<0.01$, calc. $F=4.8$ on $2,96 d f$ ). In contrast, infusion of bilirubin or control solutions, and disruption of the BBB under each experimental state had no significant effect on brain intracellular $\mathrm{pH}$ as shown in Figure 4.

The values for cerebral hemisphere bilirubin concentration are shown in Figure 5. After right-sided BBB opening a predominantly unilateral deposition of bilirubin was demonstrated. The results also show a variability in the degree of opening, and suggest a failure of opening in one experiment. The cerebral hemisphere bilirubin estimation results for two of the albumin infusion control experiments and three studies using hyperbilirubinemia alone are included for comparison. The relationship between right cerebral hemisphere bilirubin concentrations and changes seen in the brain energy status of individual animals is shown in Figure 6. A significant correlation was demonstrated between cerebral hemisphere bilirubin content and the degree of fall in $\mathrm{PCr} /(\mathrm{PCr}+\mathrm{Pi})(r=0.84, p<0.01, n=9)$. No correlation was found between serum bilirubin concentration and bilirubin concentration or changes in $\mathrm{PCr} /(\mathrm{PCr}+\mathrm{Pi})$ in the cerebral hemispheres.

The three control experiments (results not shown) in which systemic infusion was omitted and unilateral carotid perfusion was performed using isotonic saline, followed $30 \mathrm{~min}$ later by isotonic arabinose, were not associated with any detectable effect on cerebral energy status.

\section{DISCUSSION}

Phosphorus magnetic resonance spectroscopy is now well established as a method which allows sequential measurements in vivo of the relative concentrations in the brain of compounds containing mobile phosphorus nuclei. In addition, it also allows measurement of brain pHi. Several limitations of the technique must, however, be recognized, especially with regard to quantification of the data obtained. Absolute quantitation of metabolites is not possible, and uncertainties arise in determining peak areas to provide quantitation in relative terms. These issues,

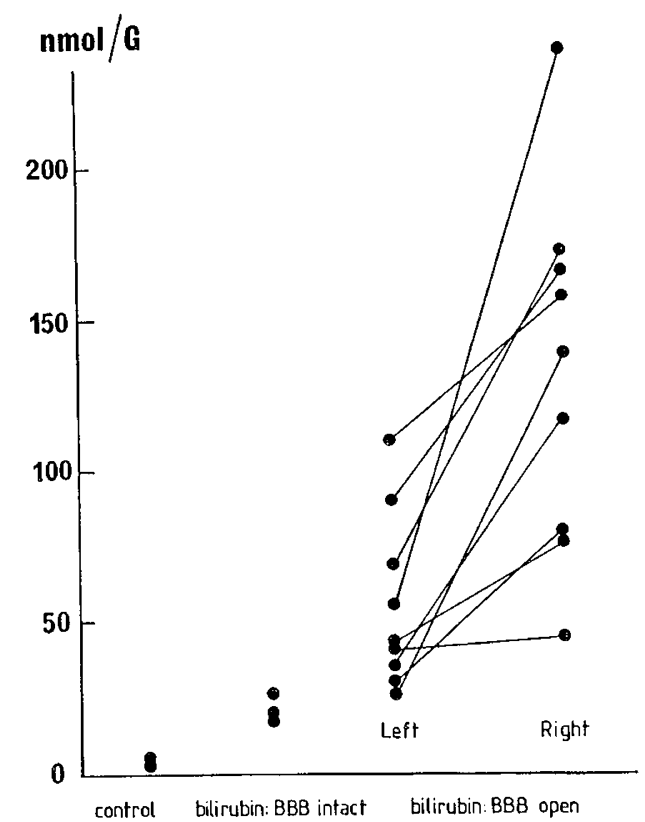

Fig. 5. Cerebral hemisphere bilirubin concentration in $\mathrm{nmol} / \mathrm{g}(\mathrm{y}$ axis) after albumin infusion (2), hyperbilirubinemia alone (3), and predominantly right-sided BBB opening in the presence of hyperbilirubinemia (9). Where right and left cerebral hemispheres were assayed separately lines link the two results from individual animals.

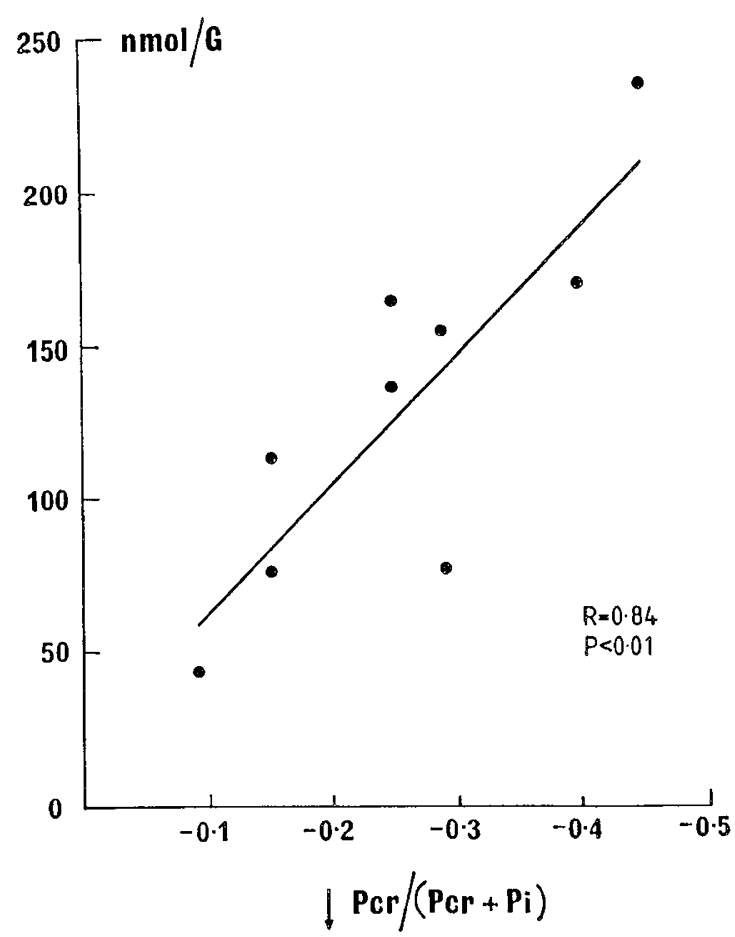

Fig. 6. Right cerebral hemisphere bilirubin concentration in $\mathrm{nmol} / \mathrm{g}$ (y axis) plotted against the fall in $\mathrm{PCr} /(\mathrm{PCr}+\mathrm{Pi}$ ) (x axis).

together with considerations relating to the determination of intracellular $\mathrm{pH}$ by this technique, have been discussed in detail elsewhere (12-14).

In addition, the precise anatomical origin of the NMRS signal is uncertain, but it can be assumed in these experiments to derive mainly from the cerebral hemisphere. Clearly, a disadvantage of this technique in relation to bilirubin encephalopathy is the current inability to achieve spatial resolution and sensitivity sufficient to allow analysis of those anatomical regions of the brain known to be especially susceptible to bilirubin neurotoxicity.

Results were expressed as the ratio of $\mathrm{PCr}$ to phosphocreatine $+\mathrm{Pi}$. This has the advantage of quantifying the reciprocal changes in $\mathrm{PCr}$ and $\mathrm{Pi}$ whereas ensuring that the variance due to noise is more consistent over a range of $\mathrm{PCr}$ and $\mathrm{Pi}$ concentrations. The ratio $\mathrm{PCr} / \mathrm{Pi}$, while theoretically a more sensitive indication of cerebral energy status, is susceptible to large errors when the denominator is small.

The technique of osmotic opening of the BBB is well established (4). The mechanism, magnitude, and time course of barrier opening and the occasional failure of hyperosmolar opening using this method has been described (15). Osmotic removal of water from capillary endothelial cells and brain parenchyma produces vasodilatation and stretching of endothelial cell membranes with an associated increase in permeability of intercellular tight junctions. The effect is reversible and may be accompanied by transient behavioural (15) or electrophysiologic (6) changes. Although associated with oedema and a decrease in regional blood flow, BBB opening is not known to be associated with any permanent pathologic changes $(15,16)$.

In these experiments, hyperosmolar opening was associated with a significant reduction in $\mathrm{PCr} /(\mathrm{PCr}+\mathrm{Pi})$ in the absence of hyperbilirubinemia. The mechanisms of this is uncertain. Control carotid infusions of isotonic saline and isotonic arabinose had no effect, indicating that it was neither a consequence of transient anoxia nor a direct effect of arabinose. The reversibility of these changes was not established, because the length of the experiment was within the anticipated duration of BBB opening documented for this method (15).

The combination of hyperbilirubinemia and unilateral hyperosmolar opening of the BBB was associated with a significant 
decrease in the ratio of $\mathrm{PCr} /(\mathrm{PCr}+\mathrm{Pi})$ in the ipsilateral cerebral hemisphere. Neither similar levels and duration of hyperbilirubinemia without barrier-opening, nor opening in the absence of hyperbilirubinemia had a comparable effect. In the experimental animals the right hemisphere was frequently stained yellow, and there was a linear correlation between brain bilirubin concentration and reduction in $\mathrm{PCr} /(\mathrm{PCr}+\mathrm{Pi})$ ratio. The significance of absolute values of brain bilirubin estimated from tissue samples is limited because they do not necessarily reflect intracellular bilirubin content. The relative values between the two hemispheres served to demonstrate a partial effect on BBB permeability in the contralateral hemisphere. This was considered to be the result of a combination of a cross-over effect of any arabinose reaching the left hemisphere, and the raised systemic serum osmolality after perfusion. Hemisphere cross-over of perfusate may occur at the circle of Willis, the extent of which is dependent on the balance between systemic blood pressure and arabinose perfusion pressure. Serum osmolality after arabinose perfusion was not measured in these experiments. Rapoport et al. (15), using the same methodology, found that serum osmolality rose transiently to over $350 \mathrm{mosmol} / \mathrm{L}$ immediately after carotid perfusion of $1.8 \mathrm{M}$ arabinose. This exceeds the threshold value of $345 \mathrm{mosmol} / \mathrm{L}$ found by Bratlid et al. (5) to be associated with increased bilirubin deposition in the rat brain.

Although it is not possible to measure "free bilirubin" in rat plasma, the molar ratio of bilirubin to albumin indicated that unbound bilirubin would have been present and entered the brain through the open $\mathrm{BBB}$ along with albumin-bound bilirubin. This was shown to be the case in a study in newborn piglets using acute urea-induced hyperosmolality (17). Previous work in the rat using a similar experimental protocol has provided evidence that the neurophysiologic effects of barrier opening in association with hyperbilirubinemia are related to the proportion of circulating bilirubin which is unbound (2).

The mechanism by which bilirubin affects brain energy metabolism is a matter for speculation. Experiments conducted in vitro have provided conflicting evidence. Early observations suggested that bilirubin inhibited respiration and uncoupled oxidative phosphorylation in mitochondria $(7,8)$, but mitochondria from whole brain or cerebellum of kernicteric guinea pigs did not demonstrate uncoupling of oxidative phosphorylation (18). Recent work in vitro using ${ }^{31} \mathrm{P}$ NMR failed to reveal any effect of bilirubin on the phosphorus spectrum of guinea pig brain slices except in combination with hypoxia (19). No change in brain intracellular $\mathrm{pH}$ was observed in the present experiments. This indicates that increased acidity in brain cells was not a factor in mediating or enhancing bilirubin neurotoxicity. Moreover, it is noteworthy that comparable degrees of reduction in brain $\mathrm{PCr} /(\mathrm{PCr}+\mathrm{Pi})$ induced by hypoxia or ischemia are accompanied by a fall in $\mathrm{pH}(20)$. In so far as this fall is mediated by lactate accumulation secondary to anaerobic glycolysis, this observation is consistent with evidence that bilirubin inhibits various enzymes of the glycolytic pathway (21). Lastly, it is possible that deleterious effects of hyperosmolar opening of the BBB, as indicated by the observation of a reduction in the $\mathrm{PCr} /(\mathrm{PCr}+$ $\mathrm{Pi}$ ) ratio, in some way render the brain more sensitive to the toxic effects of bilirubin.

The relevance of these observations to the pathophysiology of bilirubin encephalopathy in newborn infants is uncertain, but they do suggest at least one mechanism by which bilirubin may gain access to the brain, and one mechanism by which it may exert a toxic effect on brain tissue.

Theoretical considerations and experimental evidence suggest that free bilirubin crosses the BBB (22). The early idea that bilirubin was neurotoxic in the newborn because the BBB was more permeable is now known to be false (23). It is likely that at a given concentration of free bilirubin in plasma the rate of entry is greater if the barrier has been "opened." Various pathologic circumstances occur in newborns that are known to render the BBB leaky, including hypoxia, hypertension, and hyperos- molality. Unfortunately it is not possible to measure regional or total permeability of the cerebral vasculature in the human newborn. However, it seems reasonable to suppose that entry of albumin-bound bilirubin across an open barrier is not toxic, and that free bilirubin may enter the brain at rates sufficient to cause neurotoxicity through either an intact or open barrier. In adult rat cerebral hemispheres the rate of entry is clearly insufficient to cause toxic effects when the barrier is intact over the duration for which these observations were made. This does not of course exclude the possibility that free bilirubin enters at a toxic rate across an intact barrier in relevant regions of the brain of the newborn infant. In short, the two modes of entry are not mutually exclusive: free bilirubin may cross the intact barrier, and will also enter the brain through an open barrier. Either mode of entry may occur at different times, or within different regions of the brain simultaneously.

These observations provide the first evidence that bilirubin may disturb the energy metabolism of the cerebral cortex in vivo. It is likely, but unproven, that this reflects one component of bilirubin neurotoxicity in the human infant. The cerebral hemispheres are, of course, relatively spared in bilirubin encephalopathy, and it may be that a quite unrelated mechanism operates in those regions of the brain known to be peculiarly susceptible. A distinction also needs to be made between the effects of bilirubin on neuronal metabolism and function which may be reversible, and the mechanism by which bilirubin causes neuronal death. The latter does not necessarily represent the consequences of a severe or prolonged episode of the former.

Acknowledgments. The authors thank C. B. Pierce, University Data Centre, Oxford University for advice given on statistical methods, and Gail Davies for preparing the manuscript.

\section{REFERENCES}

1. Chen H, Lien I, Lu T 1965 Kernicterus in newborn rabbits. Am J Pathol 46:331-336

2. Lucey JF, Hibbard E, Behrman RE, Esquiver de Gallardo FO, Windle WF 1964 Kernicterus in asphyxiated newborn rhesus monkeys. Exp Neurol 9:43-58

3. Lending M, Slobody LB, Mestern J 1966 The relationship of hypercapnia to the production of kernicterus. Child Neurol 9:145-151

4. Levine RL, Fredericks WR, Rapoport SI 1982 Entry of bilirubin into the brain due to opening of the blood-brain barrier. Pediatrics 69:255-259

5. Bratlid D, Cashore WJ, Oh W 1983 Effect of serum hyperosmolality on opening of blood-brain barrier for bilirubin in rat brain. Pediatrics 71:909-912

6. Wennberg RP, Hance AJ 1986 Experimental bilirubin encephalopathy: importance of total bilirubin, protein binding, and blood-brain barrier. Pediatr Res 20:789-792

7. Day RL 1954 Inhibition of brain respiration in vitro by bilirubin: reversal of inhibition by various means. Am J Dis Child 88:261-264

8. Zetterstrom R, Ernster L 1956 Bilirubin, an uncoupler of oxidative phosphorylation in isolated mitochondria. Nature 178:1335-1337

9. Sawasaki Y, Yamada N, Nakajima H 1986 Developmental features of cerebellar hypoplasia and brain bilirubin levels in a mutant rat with hereditary hyperbilirubinaemia. J Neurochem 27:577-583

10. Oeff K, Konig A 1955 Das Blutvolumen einiger Rattenorgane und ihre Restblutmenge nach entbluten bzw. Durchspulung: Bestimmung mit ${ }^{32} \mathrm{P}$ markierten Erythrocyten. Arch Exp Path Pharmak 226:98-102

11. Taylor DJ, Bore PJ, Styles P, Gadian DG, Radda GK 1983 Bioenergetics of intact human muscle. $\mathrm{A}{ }^{31} \mathrm{P}$ nuclear magnetic resonance study. Mol Biol Med 1:77-94

12. Gadian DG 1982 Nuclear Magnetic Resonance and Its Application to Living Systems. Clarendon Press, Oxford

13. Hilberman M, Subramanian VH, Haselgrove J, Cone JB, Egan JW, Gyulai L, Chance B 1984 In vivo time resolved brain phosphorus nuclear magnetic resonance. J Cereb Blood Flow Metab 4:334-342

14. Cady EB, Costello AM, Chu A, Delpy DT, Gardiner RM, Hope PL, Reynolds EOR 1986 Cerebral metabolism and intracellular $\mathrm{pH}$ during hypercapnia and hypocapnia in the newborn lamb. J Physiol 328:1-14

15. Rapoport SI, Fredericks WR, Ohno K, Pettigrew KD 1980 Quantitative aspects of reversible osmotic opening of the blood-brain barrier. Am J Physiol 238:R421-R431

16. Pappius HM, Savaki HE, Fieschi C, Rapoport SI, Sokoloff L 1979 Osmotic opening of the blood brain barrier and local cerebral glucose utilization. Ann Neurol 5:211-219

17. Burgess GH, Stonestreet BS, Cashore WJ, Oh W 1985 Brain bilirubin deposition and brain blood flow during acute urea-induced hyperosmolality in 
newborn piglets. Pediatr Res 19:537-542

18. Diamond I, Schmid R 1966 Oxidative phosphorylation in experimental bilirubin encephalopathy. Science 155:1288-1289

19. Ives NK, Cox DWG, Gardiner RM, Bachelard HS 1988 The effects of bilirubin on brain energy metabolism during normoxia and hypoxia: an in vitro study using ${ }^{31} \mathrm{P}$ nuclear magnetic resonance spectroscopy. Pediatr Res 23:569-573

20. Naruse S, Horikawa Y, Tanaka C, Hirakama K, Nishikawa H, Watari H 1984 In vivo measurement of energy metabolism and the concomitant monitoring of electroencephalogram in experimental cerebral ischaemia. Brain Res 296:370-372

21. Karp WB 1978 Biochemical alterations in neonatal hyperbilirubinaemia and bilirubin encephalopathy: a review. Pediatrics 64:361-368

22. Robinson PJ, Rapoport SI 1987 Binding effect of albumin on uptake of bilirubin by brain. Pediatrics 79:553-558

23. Saunders NR 1986 Development of human blood-CSF-brain barrier. Letter. Dev Med Child Neurol 28:261-262 